\title{
Conocimiento práctico y conocimiento académico en los profesores del nivel inicial ${ }^{1}$ (seis preguntas)
}

\author{
Everyday vs academic knowledge among early \\ childhood teachers \\ (six questions)
}

Rosa Julia Guzmán Rodríguez ${ }^{2}$ Inés Ecima ${ }^{3}$

\section{Resumen}

El presente artículo, producto de un proceso de investigación cualitativa adelantado con profesoras del nivel inicial, quienes contaban con diferentes niveles de formación y trabajaban en colegios con diversas características, se centra en la reflexión sobre las formas en que aprenden a enseñar a leer y a escribir a los niños. Se encontró que independientemente del nivel de formación que tengan las profesoras, su principal fuente de aprendizaje son las prácticas. A partir de estos hallazgos se plantean algunas preguntas sobre la relación entre la teoría y las prácticas cotidianas en la formación docente y lo que esto sugiere a las instituciones formadoras de maestros.

Palabras clave

Alfabetización inicial, formación docente, conocimiento práctico, conocimiento académico.

\section{Abstract}

This paper, the result of a qualitative research process, serves as a reflection on the ways in which early childhood teachers who belong to schools with different characteristics and possess different levels of training, learn to teach reading and writing to boys and girls. It was found that, independent of their level of training and education, practice in the classroom served as their main source of learning. From these findings, some questions emerge about the relationship between the theory and the everyday practice in the training of educators and what this suggests for the institutions responsible for such activity.

\section{Keywords}

Teaching of early literacy, teacher training, practical knowledge, academic knowledge

Artículo recibido el 28 de febrero de 2011 y aprobado el 19 de agosto de 2011

1 Este artículo es producto de la investigación Cómo aprenden los educadores del nivel inicial a diseñar estrategias que faciliten la alfabetización inicial en la línea de Infancia, que hace parte del grupo de investigación EDUCACIÓN Y EDUCADORES de la Universidad de La Sabana. Fue financiado por la Dirección de Investigación de la Universidad de La Sabana. Colombia. (2008-2010) Código EDU-22-2008

2 Doctora en Educación, Universidad de Nova. Directora Maestría en Pedagogía, Universidad de La Sabana, Colombia. Correo electrónico: rosa.guzman@unisabana.edu.co

3 Magister en Educación, Universidad Pedagógica Nacional. Profesora Facultad de Educación, Universidad de La Sabana Colombia. Correo electrónico: ines.ecima@unisabana.edu.co 
«Las mentes de nosotras están muy limitadas.» (Testimonio de profesora).

«Yo digo, la universidad para uno fue más teórica, más de conocer, más de leer, pero la parte de llegar a ser uno docente es la parte práctica» (Testimonio de profesora).

«Además, es que en la universidad le presentan ciertos parámetros internos; cuando tú sales al mundo exterior a enfrentarte a los niños... es totalmente diferente» (Testimonio de profesora)

Las anteriores afirmaciones hechas por educadoras participantes en una investigación titulada Cómo aprenden los educadores del nivel inicial a diseñar estrategias que faciliten la alfabetización inicial, adelantada por la Facultad de Educación de la Universidad de La Sabana en Colombia, plantean una interesante reflexión en torno a las formas en que resulta más conveniente formar a los educadores.

En este documento se exponen algunas ideas en este sentido, producto de los hallazgos hechos en la investigación arriba mencionada, con respecto a la alfabetización inicial. Se trabajó el tema de la alfabetización, dado que el acceso a la escuela no la garantiza, y no alfabetizarse es una condición de marginalidad social muy fuerte. Es así como en el informe presentado por el PNUD (2010), se plantea un enfoque de desarrollo humano que pone el énfasis en medir dicho desarrollo en términos de la ampliación de las capacidades de las personas, entre las que se considera muy importante la alfabetización.

En este informe del PNUD se afirma que quien no disponga de dichas capacidades está condenado al estancamiento y a la exclusión. De la misma forma, en otro documento que se ocupa de este tema, se afirma: «La alfabetización, más que un objetivo en sí mismo, debe ser entendida como "la puerta" que permite a los seres humanos acceder a un universo de conocimiento, no el único, pero sí el más complejo, que viene vehiculado a través de la cultura escrita, teniendo siempre muy en cuenta que el objetivo no es aprender a leer y a escribir mecánicamente, sino incorporar la cultura lectora. Y esta puerta debería permanecer abierta en cualquier momento de la vida» (Fernández, 2007, p. 27).

Por su parte, la CEPAL (2005) afirma que hay suficientes argumentos de tipo económico que ha- cen que la alfabetización resulte rentable; entre ellos señala que la alfabetización mejora los rendimientos productivos de trabajadores y disminuye el índice de siniestralidad laboral. Estas razones, entre otras de orden pedagógico, fueron las que motivaron el desarrollo de la investigación, sobre la que aquí se presentan resultados y comentarios.

\section{La situación}

Actualmente hay muchísimos niños en el mundo que no se han alfabetizado y que, por múltiples razones, no cuentan con las condiciones para lograrlo. Una de ellas es la ineficacia de la escuela para desarrollar procesos de alfabetización en su sentido más profundo. Así se expone en un documento de UNESCO (2006), en el que se afirma que la falta de eficacia y de eficiencia de los sistemas educativos es uno de los principales problemas que se presentan en los países pobres, lo que se ve reflejado en los altos índices de ausentismo y fracaso escolar. En este documento se plantea que el fracaso escolar les cuesta a los países latinoamericanos 11.000 millones de dólares al año.

Al respecto, Rosa María Torres, a partir de un estudio mundial en el que toma como fuente de información un estudio de UNESCO OREALC (1992), aporta datos muy preocupantes, al afirmar que alrededor de la mitad de los estudiantes repite el primer grado debido a problemas en la enseñanza de la lectura y la escritura. Afirma Torres que en sectores muy pobres esta cifra puede llegar hasta el $60 \%$.

Para el caso concreto de Bogotá, de acuerdo con los datos expuestos en el documento de Lineamientos. Respuestas grandes para grandes pequeños (2006), en el año 2004, 1949 niños abandonaron la escuela en el primer grado de educación básica, y en el año 2006, 2488 niños fueron sometidos a repetir el año. El mismo documento presenta, entre las causas internas del sistema que favorecen esta preocupante situación, el no saber leer y escribir como se espera tradicionalmente.

Tanto la alfabetización inicial como el aprendizaje en general, están fuertemente determinados por las prácticas educativas que se dan cotidianamente en la escuela. Por prácticas educativas se entienden 
las acciones que se proponen en el marco de la educación, cargadas de una intención formadora hacia la población infantil. Esas acciones ocurren en procesos de interacción y en el aula de clase, entendida en un sentido amplio, como todo ese espacio físico y simbólico que configura el trabajo en las instituciones encargadas de esta función educadora.

Es necesario hacer la precisión anterior, dado que también se detectaron causas relacionadas con el tipo de actividades que se adelantan y el trato que se da a los niños en el preescolar, que permiten pensar en la necesidad de redimensionar el trabajo que se lleva a cabo en este nivel, en dos sentidos: uno, valorar lo que allí se hace, y el otro, sacar mayor provecho a las actividades desarrolladas con los niños, pues según se afirma en el documento de la SED (2006), se considera, de acuerdo con la tradición, que los niños pueden pasar su tiempo jugando en el preescolar, sin prestar mayor atención a su desarrollo cognitivo, concentrándose preferencialmente en actividades de aprestamiento que los lleven a tener una mejor coordinación fina; es decir, se asume desde la tradición que la lectura es una técnica que se mecaniza con la práctica y que la escritura es un ejercicio manual.

Los datos anteriores son motivo suficiente para indagar qué sucede en el contexto escolar con respecto al éxito que se busca en la alfabetización. Resulta preocupante constatar que los avances en la ampliación de cobertura en la educación inicial en la región, aunque son importantes, no son suficientes todavía para garantizar el acceso al que tienen derecho los niños, pero resulta aun más preocupante que quienes tienen acceso a ella tampoco logren alfabetizarse en sentido pleno.

Son varios los aspectos a tener en cuenta en este proceso. En primer lugar, como ya se ha mencionado en este documento, no todos los niños acceden a la escuela. En segundo lugar, no todos reciben una educación de calidad; y en tercer lugar, para lograr la alfabetización en sentido pleno, es necesario reconocer que esta ni empieza ni termina en el primer grado de escolaridad. Estos planteamientos hacen que sea necesario volver la mirada sobre los profesores y sus conocimientos, ideas y prácticas acerca de lo que es enseñar a leer y a escribir, así como de lo que implica apoyar la alfabetización escolar inicial.

Aquí, es necesario aclarar que entendemos la persona alfabetizada, tal como la define la UNESCO:

Está funcionalmente alfabetizada la persona que es capaz de realizar todas las actividades en que la alfabetización es necesaria para la actuación eficaz en su grupo o comunidad y que le permiten seguir valiéndose de la lectura, la escritura y la aritmética al servicio de su propio desarrollo y el de la comunidad (Fernández, A., 2007).

Sin embargo, en esta investigación se aludió siempre a la fase de alfabetización inicial, lo que significa ayudar a los niños y niñas a adentrarse en la lectura y la escritura como objetos sociales y culturales, lo que marca distancia con la iniciación tradicional a la lectura y la escritura, que la organiza basada en procesos que buscan solamente desarrollar aspectos perceptivos y motores, bajo el supuesto de que se escribe con la mano y se lee con los ojos, desconociendo así tanto la participación del cerebro, como la funcionalidad social de la lectura y la escritura.

Para referirnos a la alfabetización inicial es importante señalar que este proceso tiene su génesis en el entorno social en general y que se inicia antes de ingresar a la escuela, puesto que no es un proceso exclusivo del ámbito escolar. Esto tiene fuertes implicaciones en las condiciones de equidad en la educación de los niños, en tanto muchos de ellos, que llenan las cifras arriba citadas, provienen de contextos en los que la lectura y la escritura no constituyen prácticas frecuentes de uso social.

Por otra parte, la enseñanza tradicional desconoce estos contextos de desarrollo de los estudiantes y empieza su proceso desde supuestos erróneos (Ferreiro y Teberosky, 1979, Ferreiro y Gómez, 1982, Ferreiro 1998, Lerner 2001, Rincón 2003, entre otros), como por ejemplo:

- Considerar que todos los niños deben aprender lo mismo, dado que tienen la misma edad.

- Creer que la lectura y la escritura son procesos perceptuales que se aprenden con la imitación y la repetición. 
- Considerar que este proceso es un requisito académico.

- Pensar que la alfabetización es una cuestión que se agota en la selección de un método de enseñanza.

- Asumir que el curso en que se inicia este proceso en la escuela, debe estar bajo la dirección del profesor más inexperto o del que merece ser castigado.

- Considerar que cuando alguien no aprende a leer y a escribir es porque tiene un problema de aprendizaje.

- Considerar que los saberes que traen los niños de algunos contextos, se constituyen en un obstáculo para aprender.

Afirma Rosa María Torres (1995) en un documento de UNICEF, que el analfabetismo con frecuencia se asocia con la falta de acceso a la escuela, pero esto no siempre es así, ya que también es producto de una educación de mala calidad. Señala Torres, que es así que la alfabetización, a pesar de ser la misión más importante que la sociedad le ha encargado a la escuela, sigue siendo una misión en crisis.

En el mismo documento, Torres (1995) resalta la importancia de abordar esta temática de una manera convergente desde las políticas de atención a los niños, niñas, familias y comunidades, de tal manera que la lectura y la escritura sean fuente de disfrute y de desarrollo permanente, mediante el acceso a medios impresos, a la informática y a las bibliotecas.

Se sabe que el nivel de formación alcanzado por los padres -y especialmente por la madre- es un factor que influye de manera determinante en los resultados de aprendizaje de los hijos. Pero la formación de los docentes y los usos sociales de la lectura y la escritura que ellos tengan, también inciden de manera directa en los aprendizajes de sus estudiantes, y si estamos ante una situación en que ni los padres ni los profesores tienen las condiciones para alfabetizar a los niños adecuadamente, estamos ante un panorama bastante preocupante.

Los niños que no pueden alfabetizarse oportunamente constituyen un grupo poblacional con altas probabilidades de marginación, ya que a pesar del auge de los medios audiovisuales, la lectura y la escritura siguen siendo necesarias en el desarrollo y transmisión del conocimiento; además se requieren para acceder con propiedad al uso de los computadores. Limitar el acceso a ellos, contribuye a aumentar la inequidad. No en vano, a partir de un estudio de la Oficina Regional para América Latina de la UNESCO, se considera que se requieren al menos 6 o 7 años de escolaridad para aprender a leer y a escribir con sentido (Infante, 2000) y 12 años para dominar ambos procesos. Además, se requiere del uso de dichos procesos en diferentes contextos. Es decir, para alfabetizarse es necesario hacer un real uso social de la lectura y la escritura.

Los países latinoamericanos vienen haciendo esfuerzos importantes por ampliar la cobertura escolar; sin embargo, en la escuela se sigue presentando el fracaso como algo natural e inherente a ella, cuya responsabilidad recae en los niños y en sus familias. De una manera preocupante este fracaso se sitúa con un acento muy marcado en los primeros grados de escolaridad, donde se ha visto que el aprendizaje, tanto de la lectura como de la escritura, juega un papel muy importante en las decisiones que toman los docentes con respecto a la promoción o repetición de un año.

Por otra parte, los sistemas educativos han puesto en marcha estrategias tendientes a aumentar los años de permanencia de los estudiantes en la escuela mediante la promoción automática, por ejemplo. Sin embargo, en investigaciones adelantadas en Colombia por la Facultad de Educación de la Universidad de La Sabana, hemos encontrado estudiantes que cursan cuarto y quinto grado que no pueden escribir ni siquiera una palabra como mamá, y que plantean hipótesis sobre la construcción de la lengua escrita similares a las que puede plantear un niño alrededor de los 5 años, asista o no a la escuela. Lo anterior llama la atención sobre la importancia de atender a la escuela no solamente desde las políticas de cobertura y retención, sino desde las políticas de calidad que, por supuesto, implican a los profesores.

En este documento se pretende aportar elementos que contribuyan a comprender cómo aprenden los educadores de la infancia a generar estrategias 
que faciliten y promuevan la alfabetización inicial de los niños en interacción con su entorno. Es necesario comprender de qué maneras logran aprender los educadores y qué es lo que determina que un educador aprenda lo que aprende, ya que este proceso resulta desconocido, en gran medida, por quienes se encargan de la formación de los educadores, lo que hace que sea difícil prever el éxito de las estrategias seleccionadas para formarlos.

Explica Torres (1995) cómo los problemas de enseñanza de la lectura y la escritura están relacionados con comprensiones estrechas acerca de lo que es el lenguaje y de lo que es el aprendizaje infantil, y con la aplicación de métodos pedagógicos obsoletos. Señala además que el rendimiento de los estudiantes en lectura y escritura es un factor que pesa mucho para el docente al momento de tomar la decisión de promoverlos o retenerlos en el primer grado.

Esta situación ha determinado, durante muchos años, que existan altos índices de repitencia y deserción escolar acentuados en la población más vulnerable, con los consiguientes costos económicos y sociales, aspecto que las Facultades de Educación y otras instituciones están llamadas a analizar para buscar mecanismos tendientes a aliviar esta situación.

\section{La investigación}

\section{Metodología}

Se trabajó con 25 educadoras de diferentes contextos y recorridos de formación: madres comunitarias, estudiantes de Pedagogía Infantil, profesoras de jardín, transición y primer grado de educación básica que laboraban en el sector oficial y en el sector privado, con profesoras de instituciones educativas dirigidas al nivel preescolar y también a toda la educación básica y media.

Con todas ellas se hicieron indagaciones iniciales tendientes a identificar sus ideas con respecto a quién es un niño, qué es leer, qué es escribir, qué es alfabetizarse y cómo aprenden los infantes a leer y a escribir, así como sus concepciones y su conocimiento y manejo de los métodos de enseñanza de la lectura y la escritura.
Posteriormente, se llevó a cabo un proceso de formación durante 8 meses con ellas, respecto a la facilitación de la alfabetización inicial ${ }^{4}$, la lectura y la escritura en los niños, que incluyó el desarrollo de talleres, el comentario de videos relacionados con este tema, el comentario de clases desarrolladas por ellas y grabadas en video, y el análisis, por parte de las investigadoras, de experiencias puestas en práctica por las educadoras en sus clases y presentaciones magistrales.

Se llevó a cabo este proceso de formación de las educadoras participantes durante la investigación, porque los objetivos propuestos fueron caracterizar las maneras como aprenden, identificar cómo modifican sus ideas y cómo transforman sus prácticas para generar estrategias didácticas que faciliten la alfabetización inicial, a partir de su participación en un programa de formación en el área del alfabetismo inicial.

La información recogida se analizó, lo que permitió construir categorías emergentes a partir de las cuales se interpretaron los resultados, que fueron sometidos a procesos de lectura y relectura para extraer de ellos, en forma jerarquizada, temas, categorías, relaciones entre categorías y comprensiones.

\section{Hallazgos de investigación y preguntas derivadas}

A continuación se presentan algunas reflexiones derivadas de un proceso de investigación adelantado en la Facultad de Educación de la Universidad de La Sabana, en el que se trabajó con 25 educadoras -5 madres comunitarias y 20 profesoras-, tanto en su proceso de formación inicial, como en servicio; algunas de ellas trabajaban en el sector público y otras en el privado; unas se desempeñaban en el área urbana y otras en sectores rurales. Su nivel de formación oscilaba entre la secundaria y el postgrado, pasando por el nivel de formación universitario, y su experiencia variaba entre 3 y más de 20 años (con

4 En este contexto, facilitar la alfabetización inicial significa proponer actividades que promuevan el desarrollo del lenguaje oral y el acercamiento a los usos sociales de la lectura y la escritura en los niños. Implica, además, reconocer que este proceso no remite a prácticas como las copias o la decodificación de palabras aisladas, carentes de sentido para los niños. 
excepción de las profesoras en formación) (Guzmán, 2010). A partir de los análisis adelantados en esta investigación, es posible afirmar que las educadoras participantes en este proceso, independientemente del nivel de formación que tuvieran, para alfabetizar y para enseñar a leer y a escribir a los niños, tienen como principal fuente de sus aprendizajes las prácticas cotidianas.

Si bien las profesoras aluden a las teorías que han conocido a lo largo de su carrera universitaria o de los cursos de formación -para el caso de las madres comunitarias-, dichas teorías no explican sus prácticas cotidianas ni les sirven para resolver las situaciones que se les presentan a diario. Una de las razones puede ser que las teorías que han aprendido hablan de niños que son abordados desde una perspectiva simple y fragmentada, en la que procesos como la percepción auditiva, visual y táctil, la motricidad, el esquema corporal y el manejo del espacio, por ejemplo, se entienden aisladamente, sin que se integren en un ser completo y social, ubicado en un contexto determinado.

Las profesoras no hacen ninguna alusión a los aspectos cognitivos y lingüísticos, que guardan estrecha relación con los contextos de procedencia de los niños. Los procesos de alfabetización inicial, lectura y escritura, siguen viéndose como procesos escolares desvinculados de lo social, tanto en su origen, como en sus propósitos y fines.

Como se mencionó anteriormente, se percibe en las educadoras cierto afán por hacer alusiones a la teoría, aunque no explique lo que hacen ni sea consistente con las prácticas. Ante esta situación, surge la primera pregunta con respecto a los aprendizajes académicos y los prácticos: ¿existe una sobrevaloración de la teoría que se recibe en la formación académica, que impide que se puedan ver de manera más analítica y crítica las prácticas cotidianas? Las concepciones, entendidas como

un proceso personal por el cual un individuo estructura su saber a medida que integra sus conocimientos. Este saber se elabora, en la mayoría de los casos, durante un periodo bastante amplio de la vida, a partir de su arqueología, es decir de la acción cultural parental, de la práctica social del niño en la escuela, de la influencia de los diversos medios de comunicación $y$, más tarde, de la actividad profesional y social del adulto. Las concepciones personales son la "única trama de lectura" a la que se puede apelar cuando se confronta con la realidad (Zimmerman, 2000),

sugieren que es posible sustentar prácticas que guardan coherencia con las concepciones que tienen las profesoras de lo que es leer, lo que es escribir, lo que es enseñar y lo que es aprender, pero que resultan inadecuadas. Por ejemplo, como afirmaron varias de las profesoras, si «las niñas y los niños absorben todo lo que se les da», para ellas puede resultar lógico que se les pueda enseñar por medio de copias y de planas.

De la misma manera, estas concepciones permiten sustentar las prácticas cotidianas basadas en métodos tradicionales de enseñanza de lectura y escritura que acuden fundamentalmente a aspectos perceptivos y motores, ya que según afirman algunas docentes, «para facilitarles el aprendizaje de la lectura y la escritura hay que mostrarles letras grandes para que las vean bien y ponerlos a que hagan picado sobre las letras para que las reconozcan».

Vale la pena resaltar que a pesar de que las profesoras sienten cierta seguridad en el uso de estos métodos, se evidencia algún inconformismo con los resultados de su aplicación, ya que ellas mismas plantean que cuando alguno de los métodos no funciona, empiezan a mezclarlos para obtener mejores resultados. Aquí hay una fuente de aprendizaje muy interesante, en tanto de alguna manera se basa en el análisis de la situación para tomar decisiones con respecto al trabajo cotidiano. Sin embargo esta situación no se reconoce como tal. La literatura en general se refiere a esta situación como un "eclecticismo" sin sustento.

La decisión con respecto al método de enseñanza se toma a partir de conocimientos prácticos, que informan acerca de la necesidad de hacer adecuaciones con el objetivo de resolver situaciones para las que no cuentan con recursos teóricos. Sobre este punto vale la pena reflexionar, ya que de unos años para acá, con el reconocimiento del giro 
hermenéutico, se buscan comprensiones sobre la construcción de conocimiento basado en el sentido común, entendido como:

\begin{abstract}
una forma de conocimiento colectivo de carácter práctico: a través de la construcción de representaciones, en las luchas de clasificación de la realidad, en las que se pretende poseer el monopolio del orden y la nominación, se establecen categorías que, cuando son aceptadas ampliamente, por la vía de un proceso de naturalización del orden dominante y de sus relaciones, ganan validez general (Márquez, 2008, p. 13).
\end{abstract}

Lo que usualmente conocemos como sentido común no es algo inútil. De hecho, cuando queremos llamar la atención de alguien para que actúe con cierta cordura, se le llama a apelar a su sentido común. En este proceso hay una lógica muy diferente a la académica o a la científica, pero resulta útil para resolver situaciones de la vida cotidiana. Nosotros preferimos reconocerlo como conocimiento práctico, que ha sido construido en la cotidianidad a partir de diversas fuentes, como la praxis y las conversaciones con los colegas, entre otras.

Vale la pena aclarar que el conocimiento al que se hace referencia aquí, bien podría derivarse del sentido común o bien de la acción, pero en cualquiera de los dos casos, se considera de menor categoría que el conocimiento académico o el científico. A este respecto, afirma Davini (2002, p. 30), citando a Schön (1992): «El conocimiento que proviene de la acción puede ser considerado de segunda categoría, privilegiando otra forma de conocimiento: el académico». Este mismo autor menciona que el conocimiento académico tiene la ventaja de que puede ser controlado, medible y administrado, lo que es importante para la escuela.

Desde esta perspectiva, lo que sucede con respecto a las "mezclas" de métodos que hacen los profesores y al "ensayo y error" con las actividades que plantean, podrían ser formas de resistencia y creación (con el pensamiento y la acción) frente a aquello que no responde a la vida real cotidiana que se da en la escuela.
De otro lado, al comparar las concepciones de infancia de las madres comunitarias con las de los profesores con formación académica, se evidencia una gran diferencia entre las formas de explicar los aprendizajes de los niños, así como en la manera de organizar e interpretar sus prácticas cotidianas. Así, mientras que las madres comunitarias piensan más en términos de cuidar a los niños durante el tiempo de dedicación al trabajo para que lo disfruten y no se cansen, al tiempo que tienen en cuenta su edad, las profesoras con formación académica se inclinan a pensar más en el logro de objetivos académicos, en la exigencia fuerte para alcanzar dichas metas y en encontrar diferencias marcadas entre los estudiantes de transición y primer grado de primaria. Pero quizá la diferencia más notoria que tienen los dos grupos es con respecto a los niños pobres, pues las profesoras con formación académica consideran que a ellos hay que exigirles mucho más que a los demás para que puedan aprender.

Aquí surge un segundo interrogante: ¿La formación académica de los profesores puede llegar a constituirse en un obstáculo epistemológico para comprender e interpretar la realidad circundante en su ejercicio docente, en tanto a partir de él generan marcos de interpretación universales y por lo tanto descontextualizados y ajenos a la realidad?

Surge además un tercer interrogante con respecto a la institución educativa, su organización, sus lógicas y sus dinámicas: ¿Contribuyen ellas a desplazar la atención de los profesores hacia los aspectos formales de la escuela, ignorando a los niños?

En este orden de ideas, conviene señalar que compartimos la propuesta de Márquez (2008), quien plantea la importancia de analizar los propósitos para posibilitar la comprensión de las acciones de las personas. En la investigación a que aquí se hace referencia, se encontró que el propósito común de las educadoras es promover aprendizajes en los niños, aunque la comprensión de aprendizaje es bastante disímil entre ellas, pues depende mucho de las concepciones de infancia que cada una tiene, $y$ en las que hemos encontrado -en investigaciones anteriores-que la escuela tiene una fuerte incidencia 
y contribuye a configurarlas desde sus lineamientos y políticas (Guzmán, 2008).

Asimismo, sus comprensiones de lo que es alfabetizar, leer y escribir varían y tienen relación directa con las actividades que desarrollan en las aulas de clase. Estas comprensiones, independientemente de las teorías con las que se pretenda establecer conexiones, les sustentan sus configuraciones didácticas (en el sentido en que las expone Litwin, 1997) $\mathrm{y}$ en algunos casos se las impiden.

Un cuarto interrogante surge al analizar la manera en que tanto las profesoras como las madres comunitarias piensan sus prácticas cotidianas, en tanto ignoran la pedagogía, adjetivan la didáctica y sustantivan la actividad. Si la actividad es lo sustantivo, ¿cómo pueden proyectar sus prácticas cotidianas para lograr cumplir sus propósitos? ¿Cómo pueden establecer relaciones entre la escuela y un objeto de uso social, como es la lengua escrita?

Por otra parte, las actividades planteadas por las profesoras para enseñar a leer y a escribir a sus estudiantes, tienen un propósito y una lógica interna para cumplir dicho objetivo, pero no están articuladas con las teorías que ellas verbalizan y, en muchas ocasiones, tampoco están articuladas entre sí. La definición de estas actividades depende de varios aspectos: las ideas y creencias de las profesoras, sus concepciones de infancia, las circunstancias particulares del grupo y en ocasiones de cada niño, los recursos con que cuenta, las políticas de la institución en la que trabaja y, por supuesto, sus circunstancias particulares, entre las que se destaca su propia biografía. Esto hace que sea difícil controlar totalmente la definición y puesta en práctica de las actividades.

En ese espacio de imprecisión emergen propuestas derivadas del conocimiento práctico de las profesoras, que responden -en diferentes grados- a los propósitos que se han planteado y que persiguen a través de sus experiencias cotidianas.

\section{Lógicas de trabajo en el aula}

Las lógicas que podrían inferirse del trabajo de estas educadoras son muy particulares. La presencia o ausencia de configuraciones didácticas en las clases parece obedecer a interpretaciones subjetivas que emergen más del sentido común y/o del conocimiento práctico, que del saber académico (incluso para aquellas profesoras con formación de postgrado). Vale la pena resaltar que en este documento se plantea el sentido común como una manera de organizar el conocimiento y las prácticas, diferente de aquella que proviene del saber académico y/o científico, pero que no tiene una connotación de inferioridad con respecto a él, en términos de su utilidad.

Al respecto, Márquez (2008) señala que es posible entender el sentido común como el establecimiento de categorías derivadas de las representaciones construidas para comprender la realidad, que son aceptadas ampliamente, ganando así validez general, y en esa medida puede ser reconocido como una forma de conocimiento colectivo de carácter práctico.

Este planteamiento resulta interesante para comprender el tema que nos ocupa, ya que las profesoras que participaron en esta investigación aludieron a sus prácticas cotidianas como fuente de aprendizaje. De ellas derivan categorías que podríamos llamar híbridas entre la teoría y la práctica, que les permiten comprender su quehacer y conferirle significado. Así mismo resulta muy interesante, por las preguntas a que da lugar, constatar que en el sentido común y en el saber práctico se apoyan tanto las madres comunitarias como las profesoras con formación universitaria.

Por otra parte, vale la pena indagar cómo en este sentido común juega un papel muy importante el diálogo, ya que, según afirma Ruiz,

El camino para la conformación del sentido común es la conversación, en cuanto capacidad natural de hablar de los seres humanos. Tanto la ciencia como la cultura y la sociedad viven en el elemento del lenguaje hablado, esto es, en la conversación. El lenguaje es el que articula nuestra experiencia del mundo (Ruiz, citado por Márquez, 2008, p.p. 21-22).

Si se tiene en cuenta que a partir del intercambio de comentarios y opiniones provenientes del sentido común es posible construir saberes, el diálogo entre profesores y de ellos con otras personas, se 
constituye en una fuente de conocimientos pedagógicos que bien vale la pena explorar. A partir de este diálogo seguramente es posible validar, consolidar o refutar prácticas cotidianas en el aula, así como negociar sentidos de las mismas. En esta perspectiva, Schön (1992, citado por Davini, 2009) hace planteamientos interesantes en tanto señala que es posible construir conocimientos a partir de la acción, que son verificables y acumulables con niveles crecientes de conciencia. Pero señala también que puede presentar dificultades, por ejemplo expresar contradicciones que no se perciben, así como el hecho de estar fuertemente ligado a quien lo dice o presentar dificultades de aplicación en otros contextos, y precisa que el conocimiento a partir de la acción, más que una cuestión científica parecería ser una cuestión social.

Surge entonces aquí la quinta pregunta: ¿En qué ámbito se sitúa la educación? ¿En el científico o en el social? ¿Cómo puede abordarse con propiedad lo social desde lo científico, ignorando las negociaciones de sentido que se dan entre los profesores, entre ellos y la institución escolar, entre los profesores, la institución escolar y las comunidades en donde tiene lugar el hecho educativo? Por otra parte, ¿cómo puede alfabetizarse una persona, si no es como un hecho social?

\section{Diferencias entre las madres comunitarias y las profesoras con formación universitaria}

A partir de los hallazgos de la investigación, es posible afirmar que existe una diferencia clara entre la manera en que las madres comunitarias explican sus prácticas cotidianas y la manera en que lo hacen las profesoras con formación universitaria. Las primeras acuden directamente a sus experiencias prácticas, mientras que las segundas intentan acomodar las razones que sustentan sus prácticas cotidianas a los marcos teóricos que adquirieron en la universidad, buscando explicaciones en ellos.

Otra diferencia radica en que las madres comunitarias estructuran sus prácticas más libremente que las profesoras, quienes lo hacen tratando de ceñirse a los métodos (asumidos como una secuen- cia de pasos) que les han enseñado. Sin embargo, se abren a nuevas posibilidades cuando constatan que el método seleccionado no les da buenos resultados y ensayan combinaciones de métodos para lograr sus propósitos.

Por otra parte, las madres comunitarias llevan a los niños a través de un proceso muy espontáneo y cercano a lo familiar, mientras que las profesoras se apoyan en lo que dicen las teorías y en el diligenciamiento de formatos establecidos para procesos como la planeación de clases, las formas de evaluar a los estudiantes y las maneras de informar a los padres acerca del rendimiento de sus hijos en el colegio, entre otros. Estas diferencias se presentan de manera sucinta en el siguiente cuadro:

\begin{tabular}{|c|c|}
\hline PROFESORAS & MADRES COMUNITARIAS \\
\hline Prima la idea de alumno. & Prima la idea de niño y niña. \\
\hline $\begin{array}{l}\text { La infancia termina hacia } \\
\text { los } 6 \text { años. }\end{array}$ & $\begin{array}{l}\text { La infancia se extiende hasta los } \\
18 \text { años, de acuerdo con la ley, } \\
\text { pero hay muchos niños que tie- } \\
\text { nen responsabilidades de adultos } \\
\text { y esto les acorta su infancia. }\end{array}$ \\
\hline $\begin{array}{l}\text { Los niños son sujetos ac- } \\
\text { tivos en el aprendizaje. }\end{array}$ & $\begin{array}{l}\text { Los niños son receptores, pero } \\
\text { aprenden más rápido que los } \\
\text { adultos. }\end{array}$ \\
\hline $\begin{array}{l}\text { A los alumnos de contex- } \\
\text { tos pobres hay que exigir- } \\
\text { les mucho. }\end{array}$ & $\begin{array}{l}\text { A los niños hay que dosificarles } \\
\text { el trabajo para que no se cansen. }\end{array}$ \\
\hline $\begin{array}{l}\text { Las exigencias para niños } \\
\text { de la misma edad deben } \\
\text { cambiar según el nivel } \\
\text { educativo. }\end{array}$ & $\begin{array}{l}\text { A los niños hay que exigirles de } \\
\text { acuerdo con su edad. }\end{array}$ \\
\hline $\begin{array}{l}\text { En preescolar se juega; } \\
\text { en primaria se trabaja. }\end{array}$ & $\begin{array}{l}\text { Los niños necesitan actividades } \\
\text { lúdicas. }\end{array}$ \\
\hline $\begin{array}{l}\text { Es importante estar ac- } \\
\text { tualizado en las teorías } \\
\text { del desarrollo infantil. }\end{array}$ & $\begin{array}{l}\text { La práctica es más importante } \\
\text { que la teoría. }\end{array}$ \\
\hline $\begin{array}{l}\text { Para hablar de los mé- } \\
\text { todos de enseñanza se } \\
\text { hace referencia directa } \\
\text { a las actividades que se } \\
\text { desarrollan con los niños, } \\
\text { pero se intenta buscarles } \\
\text { un soporte teórico. }\end{array}$ & $\begin{array}{l}\text { Para hablar de los métodos de } \\
\text { enseñanza se hace referencia } \\
\text { directa a las actividades que se } \\
\text { desarrollan con los niños. }\end{array}$ \\
\hline
\end{tabular}

Con respecto a estas ideas, Davini (2009) señala que las teorías que se enseñan en la universidad a los futuros profesores, en general se imparten de una manera universalista, sin tener en cuenta que se 
van a poner en práctica en contextos determinados. En este sentido, es importante analizar cómo los diversos contextos de trabajo de los educadores se constituyen en espacios de aprendizajes nuevos y de posibilidades de resignificar lo aprendido.

Señala Davini (2009, p. 25) que varios autores como Bateson (1998), Schön (1992) y Argyris (1999) sostienen que «el aprendizaje representa la modificación de expectativas, supuestos, percepciones o de la misma acción, como resultado de las características, interacciones y valores dominantes del contexto. Es central en estas teorizaciones, el concepto de acción como comportamiento imbuido de significado». Pero dado que los significados y los sentidos no saltan a la vista, quedamos frente a la necesidad de indagar acerca de los sentidos que las acciones y actividades tienen para cada educadora. No es suficiente con analizar las acciones y las actividades en sí mismas, de manera aislada.

Esta afirmación plantea la importancia y la necesidad de conocer cómo se configura el aprendizaje de los profesores, pues parecería ser que aunque las teorías enseñadas en su proceso de formación inicial o en servicio sean pertinentes, el ciclo de aprendizaje no estaría concluido hasta que cada profesor llegue a su contexto de trabajo y lo configure basado en las características de dicho contexto y en las condiciones particulares de sus estudiantes. Por otra parte, este aprendizaje estaría actualizándose permanentemente con respecto a las prácticas cotidianas y no necesariamente con respecto a las teorías. Un aspecto muy importante de este proceso es el afectivo, ya que las educadoras expresan la necesidad de contar con buenas condiciones -tanto personales como en los estudiantes- para que los aprendizajes se logren. Si bien tenemos que reconocer la importancia del bienestar emocional para el aprendizaje, hay que reconocer que esto es necesario pero no suficiente. En este orden de ideas, las profesoras estarían definiendo sus prácticas cotidianas basadas en condiciones necesarias -pero no suficientes- en las que el sentido de las acciones estaría más orientado por el bienestar afectivo de los niños que por su aprendizaje. Quizá de allí se deriven explicaciones de la alfabetización y del aprendizaje de la lectura y la escritura como "algo mágico", que se hace "por ensayo y error" y en la que se requiere "adivinar un poco".

La idea de la importancia del bienestar afectivo de los estudiantes como condición garante del aprendizaje de la lectura y la escritura, de alguna manera se ha anclado en las formas de pensar y de actuar de los educadores en general, a través de la cotidianidad. A este respecto, Cuartas afirma (Márquez, 2008, p. 88):

El sentido es una construcción diaria e infinita. Ni el tiempo ni el espacio de esta configuración permanente pueden ser señalados de manera perentoria, pues estamos frente a una representación que se hace, se deshace y se rehace en el espacio propio de lo cotidiano.

Surge entonces la sexta pregunta, por las formas posibles de recuperar los sentidos de las prácticas cotidianas de los profesores para facilitar una negociación de dichos sentidos (con la orientación con que lo plantea Bruner, 1984) para acceder a comprensiones que vinculen éstos con el conocimiento científico y académico producido sobre la alfabetización inicial y el aprendizaje de la lectura y la escritura, con el propósito de facilitar estos aprendizajes en los niños.

Esta es una tarea muy importante, que requiere ser abordada desde la preparación inicial de los profesores, a través de sus prácticas y en la formación en servicio de los educadores, pues el aprendizaje puede darse en diferentes niveles, determinados por la presencia o la ausencia de reflexión (Bateson, 1998; Schön, 1992 y Argyris, 1999, citados por Davini, 2009). Por supuesto, la tarea de las instituciones formadoras de docentes en su etapa inicial y durante su ejercicio es promover estas reflexiones y seguir estudiándolas. 


\section{Bibliografía}

Alcaldía de Bogotá. Secretaría de Educación. (2006). Lineamientos. Respuestas grandes para grandes pequeños. Bogotá.

Bruner, J. (1984). Acción, pensamiento y lenguaje. Madrid: Alianza Editorial.

(1991). Actos de significado. Madrid: Alianza Editorial.

CEPAL (2005). Invertir mejor para invertir más. Financiamiento y gestión de la educación en América Latina y el Caribe.

Davini, M. (2009). De aprendices a maestros. Argentina: Papers Editores.

Fernández, A. (2007). Alfabetización. Entreculturas. Disponible en http://entreculturas.org. Consultado el 15 de marzo de 2009; recuperado el 15 agosto 2011.

Guzmán, R. (2010). Informe final Proyecto Cómo aprenden los educadores de primera infancia a diseñar estrategias que faciliten la alfabetización inicial. Informe presentado a la Dirección de Investigación de la Universidad de La Sabana. Chía.

Litwin, E. (1997). Las configuraciones didácticas. Una nueva agenda para la enseñanza superior. Buenos Aires: Editorial Paidós.
Márquez, J. (2008). Lógicas, racionalidades y transformaciones del sentido común. Medellín: Universidad Nacional de Colombia.

PNUD (2010). Informe sobre Desarrollo Humano 2010. Disponible en http://hdr.undp.org/es/informes/ mundial/idh2010/capitulos/es/. Consultado el 15 de marzo de 2010.

Robinson, A. (s.f.). La alfabetización, un factor vital. Disponible en portal Unesco: http://www.unesco. org/education/en/file_download.php Consultado el 20 de agosto de 2009.

Torres, R. (s.f.). Alfabetización y aprendizaje a lo largo de toda la vida. Instituto Fronesis. Disponible en http://www.fronesis.org. Consultado el 2 de febrero de 2010.

UNESCO (2006). The social benefits of Literacy. Documento de trabajo para el informe de seguimiento de educación para todos.

Zimmerman, M. y Gerstenhaber, C. (2000). Acerca del enseñar y del aprender: una aproximación a las concepciones docentes en el nivel inicial. En N. Elichiry (s.f.). Aprendizaje de niños y maestros. Buenos Aires: Editorial Manantial. 
